\title{
Body sensor networks for monitoring performances in sports: A brief overview and some new thoughts
}

\author{
Farook Sattar*1, AKM Abdul Malek Azad², Md Sarfaraj Sikder², Md Shamsul Arefin ${ }^{2}$ \\ ${ }^{1}$ Department of Electrical \& Computer Engineering, University of Victoria, Victoria, British Columbia, Canada \\ ${ }^{2}$ CARC, Department of Electrical \& Electronic Engineering, BRAC University, Dhaka, Bangladesh
}

Received: November 11, 2018

Accepted: February 11, 2019

Online Published: March 18, 2019

DOI: $10.5430 / a i r . v 8 n 1 p 25$

URL: https://doi.org/10.5430/air.v8n1p25

\begin{abstract}
This paper aims to review on body sensor networks (BSNs) for sports from performance monitoring point of view with some new thoughts. The focus of the paper is to show that wearable sensor is more efficient than cameras in measuring sport performance and thereby video data and video based systems can be replaced by wearable sensors. Here, the current state-of-the art in BSNs are mainly introduced relating to sports performance instead of physical activity and health/safety related issues for sports and to the best of our knowledge, this has not been done yet for different types of sports rather than a particular sport. Although the progress in BSN for sports performance is in early stage, the ultimate goal is to develop a complete training/match analysis tool using wearable sensors and various analyses techniques to monitor as well as improve performances in sports.
\end{abstract}

Key Words: Body sensor network, Wearables, Sports, Performance analysis, Signal processing, Pattern recognition, Machine learning

\section{INTRODUCTION}

The recently proposed body sensor networks (BSNs) incorporate context aware sensing for increased sensitivity and specificity. To facilitate the performances in sports, the recent developments in BSN are presented here. In general, with its low power, flexible and compact design, the BSN provides a versatile environment for wireless sensing research and development. ${ }^{[1]}$ For instance, a comparison between wearable sensor and camera is shown in Ref. ${ }^{[2]}$ for performance measurement in swimming. The paper concludes that wearable sensor based measurements of swimmer's acceleration profiles have the capability to provide significant advances in coaching technique over the camera based measurements. Moreover, the importance and effectiveness of a wearable sensor for the water-skiers to reduce the lower back injuries have been investigated and explained but a measurement error has also been reported when obtaining the data using high frequency camera in Ref. ${ }^{[3]}$ Furthermore, the effectiveness and advantages of using wearable sensors over the wearable camera have been investigated in Ref. ${ }^{[4]}$ The paper highlights that using wearable cameras can cause occlusion effects, the correspondence issues, higher processing cost and storages whereas using smaller inertial sensors can be used within or beside the objects and it does not include occlusion.

In sports, BSN systems can be categorized into three groups by considering whether they are related to physical activities (e.g. limb movements during gymnastics), or health and safety (e.g. stress level during car racing), or sports performance (e.g. cycle mean velocity during swimming).

\footnotetext{
*Correspondence: Farook Sattar; Email: farook_sattar@yahoo.com.sg; Address: Department of Electrical \& Computer Engineering, University of Victoria, Victoria, British Columbia, Canada.
} 
One review on BSN related to physical activities for sports is presented in Ref., ${ }^{[5]}$ while another review on BSN referring to health and safety issues during sports is reported in Ref. ${ }^{[6]}$ This article presents the recent state-of-the-art of BSN for performance in various sports rather than a particular sport (e.g. swimming). ${ }^{[7,8]}$

\section{ILluSTRATIVE CONCEPTUAL FRAME- WORK ON BSN IN SPORTS}

In this section, an overview of BSN architecture followed by human body movement representation (i.e. the real movement of human body) with the typical sensing data in sports are illustrated. The relationship between the three inertial sensors (accelerometer, gyroscope and magnetometer) data from $\mathrm{BSN}$ and the human back movements is presented through the computation models (i.e. Euler angles, quaternions as well as rotation matrix for 3D rotations, and Kalman filtering for inertial tracking) and the measurement framework of the three inertial sensors.

\subsection{Overview of BSN architecture}

Figure 1 contains the overall architecture of the BSN network containing different types of sensors e.g. EEG sensor, visual sensor, EMG sensor, respiration sensor, ECG sensor, blood pressure sensor, temperature sensor, pressure sensor, accelerator, gyroscope, magnetometer, sink node etc., could be placed in different body parts or locations to obtain the physical data and carry out initial processing. All the data are accumulated by the sink node and then passed on to the base station and then send out to the cloud/internet so that the data can be shared on various health care systems, sports learning systems, navigation systems, augmented reality systems, industrial quality control, robotics, social welfare, patient's devices, diagnosis tools and others. ${ }^{[9]}$

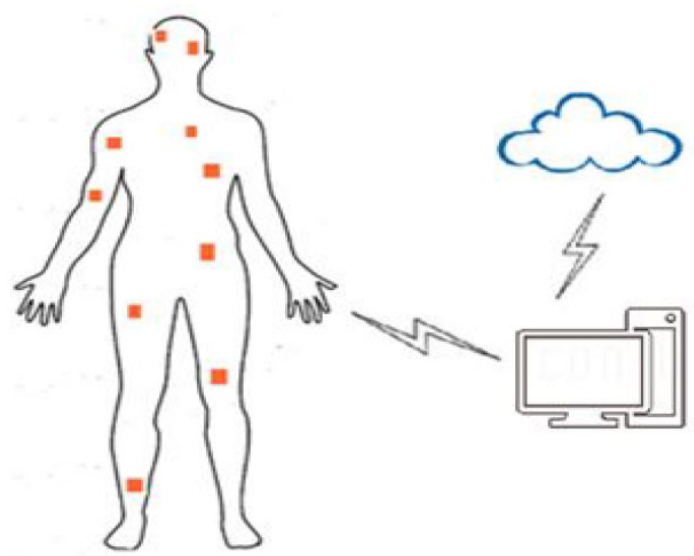

Figure 1. Overall architecture of BSN network including different types of sensors ${ }^{[9]}$

\subsection{Human back movement}

To determine the 3D bending and twist of the human back movement, Zhang et al. 2011 in Ref. ${ }^{[10]}$ proposed an updated method, where five BSN sensor nodes have been placed on the back of the human body, each containing a 3-axial accelerometer, a gyroscope and a magnetometer. This architecture in Figure 2 is used to demonstrate the motion reconstruction structure. After that, Euler angles have been identified to symbolize the orientation of back segments. Consequently, kinematics for the Euler angle has been estimated and then an unscented Kalman filter (UKF) is used to find the Euler angles.

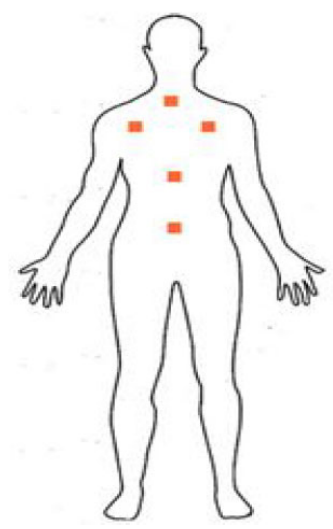

Figure 2. Placement of sensor nodes on the back of the human body [10]

\subsubsection{Euler angle}

The sensor nodes have been placed on the on the left shoulder, right shoulder, upper spine, middle spine, and lower spine, as illustrated in Figure 2. During the experiment, the prime focus has been to determine the rotational movement of the human back and hence orientation has been incorporated in the state vector. Euler angles have been chosen to define orientation of the segmentation of human back in terms of $\Phi, \theta, \Psi$ which denotes roll, pitch and yaw correspondingly, representing positive rotation in $x, y$ and $z$ axes of the body in that order. The change from the global coordinate frame to the body coordinate frame have been represented using three consecutive rotations. ${ }^{[10]}$

$$
R(\Phi, \theta, \Psi)=R_{z}(\Psi) R_{y}(\theta) R_{x}(\Phi)
$$

where,

$$
\begin{aligned}
& R_{x}(\Phi)=\left[\begin{array}{ccc}
1 & 0 & 0 \\
0 & \cos (\Phi) & -\sin (\Phi) \\
0 & \sin (\Phi) & \cos (\Phi)
\end{array}\right] \\
& R_{y}(\theta)=\left[\begin{array}{ccc}
\cos (\theta) & 0 & \sin (\theta) \\
0 & 1 & 0 \\
-\sin (\theta) & 0 & \cos (\theta)
\end{array}\right]
\end{aligned}
$$

ISSN 1927-6974 E-ISSN 1927-6982 
and

$$
R_{z}(\Psi)=\left[\begin{array}{ccc}
\cos (\Psi) & -\sin (\Psi) & 0 \\
\sin (\Psi) & \cos (\Psi) & 0 \\
0 & 0 & 1
\end{array}\right]
$$

If $\omega(t)$ is the angular rate then the Euler angle integration kinematics can be derived from the formula ${ }^{[10]}$

$$
\Gamma^{\prime}(t)=W(\Gamma(t)) \omega(t)
$$

where $W(\Gamma(t))$ is the Jacobian matrix, which is linked with the absolute rotation angle $\Gamma(t)=\left[\begin{array}{llll}\Phi(t) & \theta(t) & \Psi(t)\end{array}\right]^{T}$ to the angular rate $\omega(t)=\left[\begin{array}{lll}\omega_{x}(t) & \omega_{y}(t) & \omega_{z}(t)\end{array}\right]^{T}$. The correlation between Euler angles and the angular rate can be expressed: ${ }^{[10]}$

$$
\left[\begin{array}{c}
\omega_{x}(t) \\
\omega_{y}(t) \\
\omega_{z}(t)
\end{array}\right]=\left[\begin{array}{c}
\Phi^{\prime}(t) \\
0 \\
0
\end{array}\right]+R_{x}(\Phi(t))\left[\begin{array}{c}
0 \\
\theta^{\prime}(t) \\
0
\end{array}\right]+R_{x}(\Phi(t)) R_{y}(\theta(t))\left[\begin{array}{c}
0 \\
0 \\
\Psi^{\prime}(t)
\end{array}\right]
$$

and then we can get:

$$
W(\Gamma(t))=\left[\begin{array}{ccc}
1 & \sin (\Phi(t)) \tan (\theta(t)) & \cos (\Phi(t)) \tan (\theta(t)) \\
0 & \cos (\Phi(t)) & -\sin (\Phi(t)) \\
0 & \sin (\Phi) / \cos (\theta(t)) & \cos (\Phi(t)) / \cos (\theta(t))
\end{array}\right]
$$

\subsubsection{Quaternion}

It is one of the methods to derive the rotation matrix and in the field of mathematics it is mainly utilized for expanding the complex equations. The basic equation for the quaternion can be defined by

$$
\mathbf{q}=q_{0}+q_{1} \mathbf{i}+q_{2} \mathbf{j}+q_{3} \mathbf{k},
$$

$$
R=\left[\begin{array}{ccc}
\cos (\theta)+u_{x}^{2}(1-\cos (\theta)) & u_{x} u_{y}(1-\cos (\theta))-u_{z} \sin (\theta) & u_{x} u_{z}(1-\cos (\theta))+u_{y} \sin (\theta) \\
u_{y} u_{x}(1-\cos (\theta))+u_{z} \sin (\theta) & \cos (\theta)+u_{y}^{2}(1-\cos (\theta)) & u_{y} u_{z}(1-\cos (\theta))-u_{x} \sin (\theta) \\
u_{z} u_{x}(1-\cos (\theta))-u_{y} \sin (\theta) & u_{z} u_{y}(1-\cos (\theta))+u_{x} \sin (\theta) & \cos (\theta)+u_{z}^{2}(1-\cos (\theta))
\end{array}\right]
$$

where $R$ is an orthogonal matrix, where the inverse matrix is identical to its transpose form, such as $R R^{T}=R R^{-1}=I$ and $\mathbf{u}=\left[u_{x}, u_{y}, u_{z}\right]$ is a unit vector with $u_{x}^{2}+u_{y}^{2}+u_{z}^{2}=1$. The determinant of $R$ can be $\operatorname{defined}: \operatorname{det}(R)=1$ and three eigenvalues of $R$ can be defined as: $1, \cos (\theta)+i \sin (\theta)$ and $\cos (\theta)-i \sin (\theta)$. Further details can be found in Ref. ${ }^{[11]}$

In comparing the Euler angle, quaternions and rotation matrix; the Euler angle contains three self-governing variables and there are no redundant variables. However, the disadvantage for Euler angle is vital as it restricts smooth tracking in all directions because of the discontinuities. Although rotation matrices are suitable for computing but the nine elements of the rotation matrix contain three self-governing variables which cannot express the rotation clearly. On the other hand, quaternions can overcome the drawbacks of the Euler angle and rotation matrix. They are much easy to ap- which is a 4D vector with the above real numbers, $\mathbf{i}^{2}=\mathbf{j}^{2}=\mathbf{k}^{2}=$ $\mathbf{i j k} \mathbf{k}=-1$, and $\|\mathbf{q}\|_{2}=\sqrt{q_{0}^{2}+q_{1}^{2}+q_{2}^{2}+q_{3}^{2}}=1$. It is to be noted that, quaternion equation can be defined in terms of Euler angle equations. Further details on quaternion can be found in Ref. ${ }^{[11]}$

\subsubsection{Rotation matrix}

A rotation matrix is expressed in terms of a matrix to complete a rotation in Euclidean space. The rotation matrix for the $2 \mathrm{D}$ space can be expressed ${ }^{[11]}$ as

$$
R(\theta)=\left[\begin{array}{cc}
\cos (\theta) & -\sin (\theta) \\
\sin (\theta) & \cos (\theta)
\end{array}\right]
$$

For the $3 \mathrm{D}$ space, the equation can be expressed ${ }^{[11]}$ as ply and elude the gimbal lock. Moreover, they have better numerical steadiness than the rotation matrices and contains only four elements. As a result, recent studies utilize quatenions to execute numerical analysis in computer graphics and motion analysis to represent the rotation in $3 \mathrm{D}$ space. ${ }^{[11]}$

\subsubsection{Measurement framework}

The wearable sensor unit offers three types of measurements, such as acceleration, magnetic field and angular rate. The basic measurement equation $h$ can be defined by the following expression: ${ }^{[10]}$

$$
z_{t}=\left[\begin{array}{c}
z_{t}^{a} \\
z_{t}^{m} \\
z_{t}^{g}
\end{array}\right]=h\left(x_{t}\right)+v_{t}=h\left(x_{t}\right)+\left[\begin{array}{c}
v_{t}^{a} \\
v_{t}^{m} \\
v_{t}^{g}
\end{array}\right]
$$

where, $x_{t}=\left[\begin{array}{ll}\Gamma(t) & \omega(t)\end{array}\right]^{T}$ with $\Gamma(t)=\left[\begin{array}{lll}\Phi(t) & \theta(t) & \Psi(t)\end{array}\right]^{T}$, $\omega(t)=\left[\begin{array}{lll}\omega_{x}(t) & \omega_{y}(t) & \omega_{z}(t)\end{array}\right]^{T}, v_{t}$ is considered to be zero- 
mean additive white Gaussian noise with covariance matrix $\mathbf{V}$, as well as $z_{t}^{a}, z_{t}^{m}$, and $z_{t}^{g}$ are the acceleration, magnetic field and angular rate measurements, respectively.

Since the movement of the human back is fairly constant, the three axis accelerometer has been used to determine the gravity field vector relating to the global coordinate system. By characterizing $g=\left[\begin{array}{lll}g_{x} & g_{y} & g_{z}\end{array}\right]^{T}$ as the vector of the gravitational field determined in the global coordinate framework, the final acceleration equation can be defined as Ref. ${ }^{[10]}$

$$
z_{t}^{a}=\left[\begin{array}{c}
z_{t}^{a, x} \\
z_{t}^{a, y} \\
z_{t}^{a, z}
\end{array}\right]=R_{s}^{b} R(\Phi(t), \theta(t), \Psi(t)) g+v_{t}^{a}
$$

where $v_{t}^{a}$ denotes the acceleration measurement noise, $R_{s}^{b}$ stands for the offset for sensor bias.

The magnetometer determines the magnetic field and it can be defined by the conversion of the global magnetic field to the local sensor coordinate frame. The magnetometer measurement formula is very similar to the accelerometer's measurement framework and it can be represented by $m=\left[m_{x}, m_{y}, m_{z}\right]^{T}$ as the vector of the magnetic field determined in the global coordinate framework. The final magnetometer's equation can be defined by Ref. ${ }^{[10]}$

$$
z_{t}^{m}=\left[\begin{array}{c}
z_{t}^{m, x} \\
z_{t}^{m, y} \\
z_{t}^{m, z}
\end{array}\right]=R_{s}^{b} R(\Phi(t), \theta(t), \Psi(t)) m+v_{t}^{m}
$$

where $v_{t}^{m}$ denotes the magnetic measurement noise.

Subsequently, gyroscopes are used to estimate the angular velocity in the local coordinate system for the sensors. The angular velocity $\omega(t)$ is incorporated in state vector and hence the final gyroscope equation can be defined as Ref. ${ }^{[10]}$

$$
z_{t}^{g}=\left[\begin{array}{c}
z_{t}^{g, x} \\
z_{t}^{g, y} \\
z_{t}^{g, z}
\end{array}\right]=R_{s}^{b} H x_{t}+v_{t}^{g}
$$

where $H=\left[\begin{array}{ll}0 & I_{3 \times 3}\end{array}\right]$ and $v_{t}^{g}$ denotes the angular rate measurement noise.

\subsubsection{Kalman filtering}

Usually, the Kalman filter works on a probability distribution of state vector, which can be represented by the first and second order statistical parameters, such as mean and covariance. However, a limiting factor for the Kalman filter is that, it can only handle linear and Gaussian factors but the measurement equations are non-linear. In order to solve this issue, an upgradation to the general Kalman filter, defined as UKF can be used which can solve the non-linearity as well. Further details related to the mathematical models and the estimations can be found in Ref. ${ }^{[10,11]}$ Therefore, in general, Euler angles have been determined to characterize the direc- tion of the human back, kinematics analysis for the Euler angles have been done and then UKF has been further used to obtain the Euler angles.
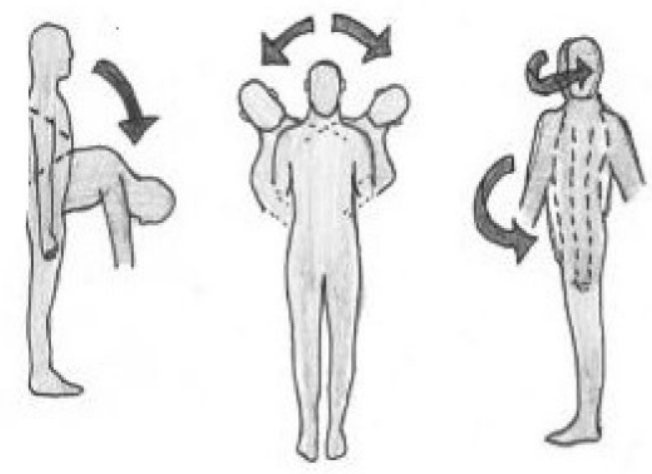

Forward Bend Side Bend Back Twist

Figure 3. The three fundamental human back movements

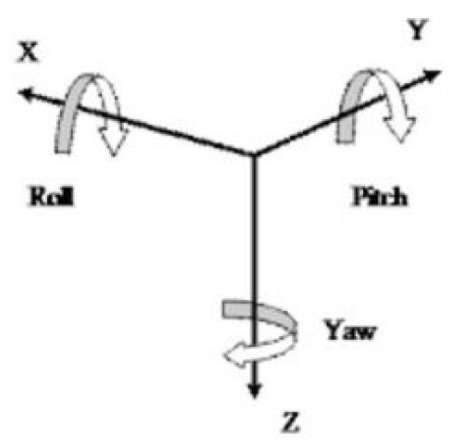

Figure 4. The global coordinate system

Using the above methods and formulas, an experimental study has been conducted to determine and investigate the Forward Bend, Back Twist and Side Bend as displayed in Figure 3. In order to perform the forward bending test, the participant has been requested to bend his body forward few times, where the rotational back movement has been in the $z$-axis in the global coordinate system (i.e. yaw angle) as shown in Figure 4. Based on the results, the roll and pitch angles for the spine and shoulders have been found to be of zero value as the participant moving forward, which reflects the real time scenario. Consequently, to perform the back twist the participant has been requested to twist his body left and right few times where the rotational back movement has been in the $x$-axis in the global coordinate system (i.e. roll angle). From the results, the pitch and yaw angles for the spine and shoulders have been found zero and it is similar to the real time situation. Similar to the previous steps, the participant has been asked to bend his body left and right few times to perform the side bending test, where the rotational back movement has been in the $y$-axis in the 
global coordinate system (i.e. pitch angle). From the results, the pitch angles for the upper spine, left and right shoulders have been the same, due to the upper part of the back shifts all together as the participant bends for the test. Also, the movement of the upper spine compared to the lower spine has been found to be much higher. ${ }^{[10]}$ Moreover, Sheng, 2012 investigated and analyzed the upper limb movements in the $3 \mathrm{D}$ space. ${ }^{[11]}$

\section{BOdy SENSOR NETWORK (BSN) FOR PERFORMANCE MONITORING IN SPORTS}

- Importance of BSN in sports:

Existing camera-based systems to determine sports performance require a complex setup and calibration procedure. In contrast, wearable (e.g. inertial ${ }^{[8]}$ ) sensor-based methods can provide similar information with a low-cost and easy maintainable sensor setup. For example, inertial sensors, such as accelerometer, gyroscope, magnetometer, provide both low- and high-order parameters in performance monitoring for sports. ${ }^{[8]}$

- General framework for BSN in sports:

The general structure of the BSN in sports is shown in Figure 5, where the role of BSN in the performance analysis for sports is highlighted. The performance monitoring in sports by wearable sensors is involved with perfornance quantification, performance assessment as well as performance prediction as indicated in Figure 5. The two other roles of BSN in sports are related to physical activities (such as running, walking, etc.) as well as health and safety (such as heart rate variability, fatigue, etc.) which are not the scope of this paper.

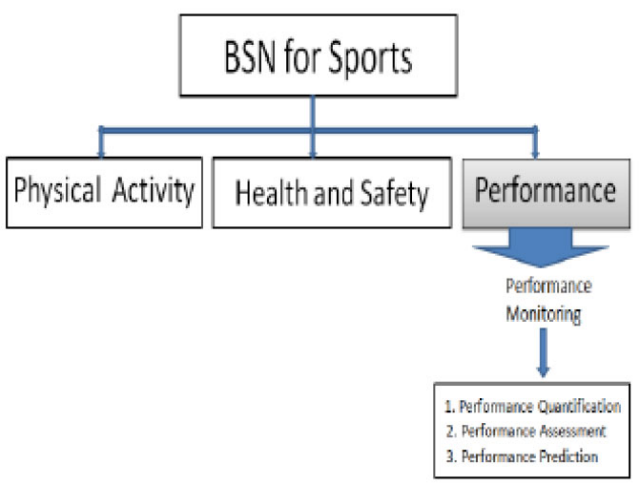

Figure 5. The general framework for BSN in sports

\section{RECENT DEVELOPMENTS ON BSN FOR SPORTS PERFORMANCE}

In Ref., ${ }^{[12]}$ three EXLs3 IMU sensors (each sensor consists of 3-axis accelerometer, 3-axis gyroscope and 3-axis magnetometer working at sampling rate of up to $200 \mathrm{~Hz}$ ) are used and they are integrated with the tennis racket as well as left and right shoes of a tennis player. In order to do the movement analysis of an individual tennis player, different types of shots (e.g. forehand and backhand with topspin and slice, smash) and footsteps (shot steps and side steps) are detected and classified during training/play. The results are obtained using a small dataset from four subjects. The detection accuracy of $76 \%$ and classification accuracy of $95 \%$ are obtained for the steps, while classification rate is reported as high as $94 \%$ for various shots. Note that a segmentation algorithm is used for detection, while classification is performed using SVM classifiers. Using this BSN system, an expert player or a trainer of an amateur player is able monitor the timing analysis of the racket as well as foot movements to improve the performance in tennis. However, the system is user dependent and needs to be robust (i.e. user independent) perhaps by using a large dataset with more subjects.

In Ref., ${ }^{[13]}$ the data from a 3 -axis accelerometer as well as a gyroscope are collected and stored to assess the swimmer's performance for $200 \mathrm{~m}$ individual medley (IM), since it has all official strokes, i.e. butterfly, backstroke, breaststroke and freestyle. The breathing patterns are adopted as follows: in butterfly, breathe every two strokes for the first $25 \mathrm{~m}$ and every stroke for the second $25 \mathrm{~m}$; in freestyle, breathe every two strokes during the first length and every three strokes in the second length of the swimming pool. The breathing data of 3 orthogonal accelerations and angular velocity components are filtered using a moving average filter with size 20 samples and can be applied to calculate the parameters such as stroke type, lap time, lap count, stroke frequency and distance per stroke, to quantify the performance of a swimmer. The block diagram of the corresponding BSN system for swimming is presented in Figure 6.

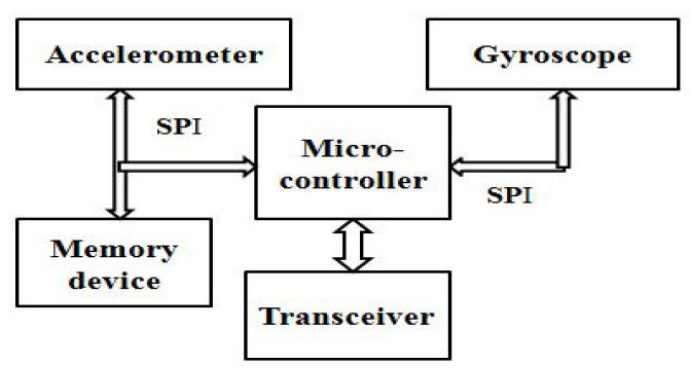

Figure 6. A system to evaluate and identify swimming parameters 
In Ref., ${ }^{[14]}$ BSN systems are presented for skating and ice hockey using three EXLs3 IMU sensors. Eight skating motions (i.e. accelerating, breaking, narrow curves, wide curves, forward-backward turns, backward-forward turns, backward skating, and jumps) are analyzed and recognized as the sensors are clipped to the shoelaces of the skate. Using acceleration and rotation velocity data, the skating motions are detected and then recognized from the features using timing characteristic and maximal acceleration for power strokes, foot's orientation at specific time. Two approaches of featured based classification and template matching are used. For classification, a sliding window with $12.5 \%$ overlapping is used on the raw data to extract different temporal (mean, standard deviation, min, max, number of zero crossings, etc.) and spectral (fast Fourier transfer coefficients) features, feature selection is done based on mutual information and Random Forest classifier is used for classification. In template matching, input three-dimensional accelerometer and gyroscope data are fused into a one-dimensional sequence using $k$-means clustering algorithm and the similarities between training template and test data is calculated using a wrapping algorithm as well as compared with a threshold to recognize different skating actions. Two sets of data are used for evaluation - one dataset $\left(S_{1}\right)$ is with an amateur skater and the second set $\left(S_{2}\right)$ consists of data from six skaters with different skills. Note that video recordings are used for conventional reference. For $S_{1}$, the overall F1 score (i.e. the weighted average of Precision and Recall, see at Wikipedia ${ }^{[15]}$ ) of all activities is found 0.92 , while it is decreased to 0.65 for $S_{2}$ most probably due to the large variations in speed and intensity among the participant skaters. The block scheme of the above mentioned BSN system for skating is depicted in Figure 7.

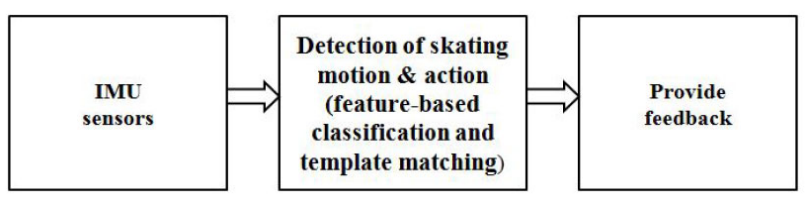

Figure 7. Simplified block scheme for detection of skating motion and actions

For ice hockey, the BSN system introduced in Ref. ${ }^{[14]}$ analyses the wrist and slap shots using sensorized hockey stick and the data from the IMU sensor (placeed at player's upper body and both wrists) which is sampled at $100 \mathrm{~Hz}$. The analysis of the recorded shots are performed in two stages: In stage one, the system detects/classifies into wrist or slap shot using a rule-based classification algorithm based on stick orientation information. In stage 2 , the system extracts a set of characteristic features for each shot, such as the max- imum, minimum and mean rotation velocities of the stick in all three axes during the shot, acceleration and timing characteristics (e.g., duration between maximum wind up and ice contact), the maximum flexion of the stick, the hand positions, etc. for training a Support Vector Machine (SVM) classifier that assesses players skill level based on their shot execution. The results are obtained using the data from 11 amateur and 8 professional players and the extracted features of the selected shots from the players are used to train a SVM classifier which can differentiate the shots for the beginners and the professional players. The Sequential Forward Selection (SFS) algorithm is used for feature selection which shows that the minimal rotation velocity in shooting direction (around $y$-axis), the duration of the pre-swing phase, and the maximum flexion of the stick, are the most relevant features for slap shots. For wrist shots, the selected features are also related to the lower hand position, the minimal and maximal rotation velocities around the $y$ - and $x$-axes, and the summed up rotation around the $x$-axis. Using leave-one-out cross validation, SVM classification algorithm gives an accuracy of $92.9 \%$ for slap shots and $88.5 \%$ for wrist shots. The block scheme of the corresponding BSN system for ice hockey is shown in Figure 8.

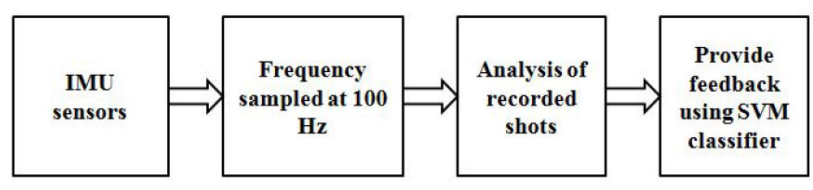

Figure 8. Monitoring shots with modified hockey stick to provide feedback to the players

Moreover, another BSN system is proposed in Ref. ${ }^{[14]}$ by integrating three IMU sensors placed on the gloves and upperbody protector of an athlete to monitor various game events (e.g. slap shots, wrist shots, hits, turns, time in motion, breaks) during the game play. Feature-based classification is performed using combined statistical and spectral features from three wearable sensors by multiple Random Forest classifiers. The acceleration and rotational velocity data from three IMU sensors and four hockey player are sampled at $100 \mathrm{~Hz}$ providing the following results: $F_{1}$ score for event detection is $70 \%$, classification rate to recognize whether a player on the ice or not is $86 \%$. In fact, due to large variations in hockey motions (specially the direction of turns) among the hockey players, the results may be deteriorated. It is expected that the results can be improved by performing training with large number of recordings during actual hockey games.

In Ref., ${ }^{[16]} 3$-axis acceleration and rotational velocity data from wearable sensors are used to characterize different 
states of a golf swing among amateur and professional golf players. Sensors are mounted in different parts of the body (left wrist, solar plexus, right knee, head) and different steps of the golf swing consist of backswing, downswing, impact, and follow-through, are featured as well as compared between a beginner and an experienced player. For example, the acceleration data of $z$-axis from solar plexus and the acceleration data of $y$-axis from wrist are found useful to differentiate the performance between the beginner and experienced players and can assist for enhancing the performance of the beginners by automatically providing advice to them. In future, it needs to incorporate machine learning tools (e.g. SVM) to automatically distinguish a beginner from an experienced player and provide more proper advice to the beginners.

In Ref., ${ }^{[17]}$ wearable sensors attached to swimmer's back (Horizontally) are used to design a biofeedback control system for freestyle swimming based on trunk rotation measurement. Four recreational swimmers have swum $200 \mathrm{~m}$ with and without feedback. A nine-degree-of-freedom inertial sensor MPU-9250 is placed horizontally to swimmers back. The instantaneous feedback on rotation status is sent to a swimmer to improve swimmers kinematics/key performance index (e.g. lap time, stroke rate) by informing the swimmer whether the medial-lateral rotation angle is $<40^{\circ}$, or within $40^{\circ}-50^{\circ}$, or $>50^{\circ}$ meaning no vibration, or one vibration, or two burst vibrations, respectively. Vibratory feedback is sent to the swimmer to adjust the roll angle (i.e. trunk rotation) if the rotation angle lies between $40^{\circ}$ and $50^{\circ}$. The results show that BSN based biofeedback mechanism improves swimmers performance by reducing average lap time by $4.5 \%$ due to increase of stroke rate and can assist a swimmer during regular training for fitness maintenance. The block diagram of the respective BSN system for swimming is depicted in Figure 9.

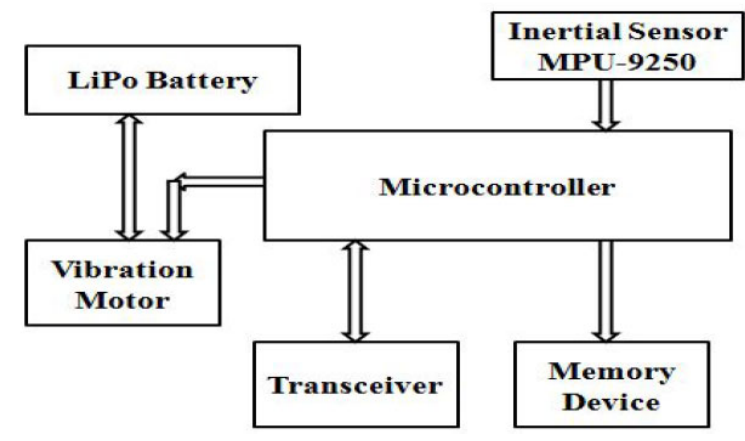

Figure 9. Wearable biofeedback control system demonstration for swimmers

In Ref., ${ }^{[18]}$ an IMU sensor is attached to the skis to determine the orientation angle (i.e. the angle of attack) during ski jumping. The angle of attack is defined by the ski orientation and the horizontal plane. For each time $t$ of the jump, the current ski orientation can be found by projecting the gyroscope data at time $t$ into $x-z$ plane and simply calculating the angle from $x$ - and $z$-components of the velocity/gyroscope data. The results obtained from 3 ski jumpers give rms errors of $2^{\circ}$ for right ski and $9.3^{\circ}$ for left ski using the video camerabased results as conventional reference. The block diagram of the respective BSN system for ski jumping is shown in Figure 10.

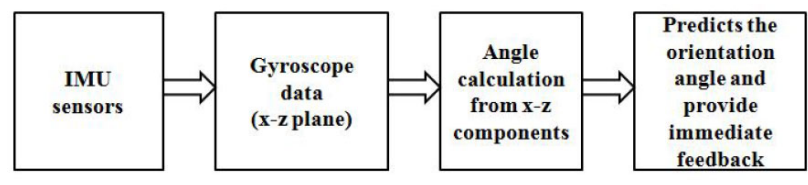

Figure 10. Block diagram for monitoring the performance of ski jumpers

In Ref., ${ }^{[19]}$ an IMMU (inertial-magnetic measurement unit) sensor is placed in the front of snowboard to classify different tricks for freestyle snowboarding using a two-stage method. In the first stage, the grind tricks are detected from high magnetometer signal variance, while air tricks are detected from low accelerometer signal variance and the remaining tricks are categorized as no-tricks or incorrectly detected tricks. In the second stage, different classifiers (e.g. Naive Bayes [NB], C4.5, k-NN, SVM) are used to classify grinds into board slide/front slide grinds and airs into broad slide/front slide airs using different rotational features obtained from the gyroscope data. Using a total of 745 tricks with different categories, the overall detection and classification accuracies are achieved for more than $90 \%$ for all categories of tricks. However, this BSN system is yet to be implemented in real-time.

In Ref., ${ }^{[20]}$ a single IMU sensor is attached to the front end of a table tennis racket handle to detect and classify various stroke types (drive, push, block and topspin for both forehand and backhand) among amateur and professional players. Kinematic data of acceleration and angular velocity are collected to detect stroke events and classify basic stroke types using pattern recognition algorithms. The strokes are detected based on the energy of the accelerometer data followed by peak detection algorithm. The stroke classification is done in the following steps: 1) annotate strokes based on stroke intervals around the detected peaks, 2) extract all stroke signals, 3) calculate 60 different features including statistical moments (mean, standard deviation, skewness, kurtosis), signal characteristics (minimum, maximum, energy, median, interquartile range), heuristic features (x-y,x-z,y-z 
correlations) for all data components, 4) classify different types of strokes using a SVM classifier. The overall mean F-measure score of $96.9 \%$ and classification rate of $96 \%$ are found for stroke detection and classification, respectively, among 10 players ( 3 professional and 7 amateur). The intent of this study is to develop a wearable device which could be attached to the body using a wristband or integrated invisibly into the table tennis racket. The block scheme of the above discussed BSN system for table tennis is presented in Figure 11.

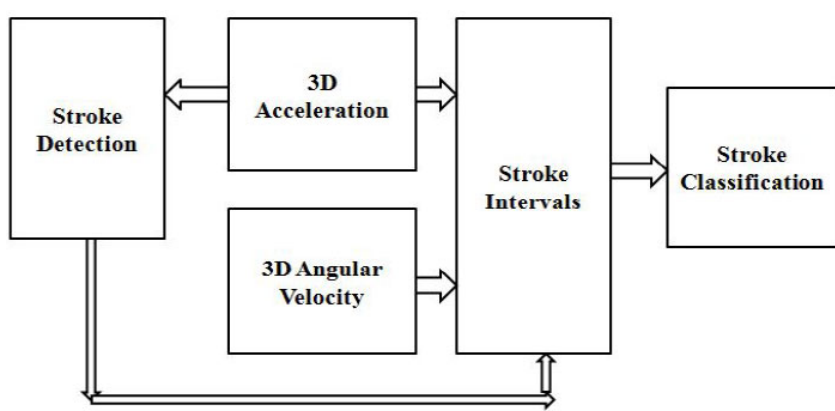

Figure 11. Simplified stroke detection system for table tennis

In Ref., ${ }^{[21]}$ the feasibility of accelerometer and GPS sensors are studied to quantify kinematics variables in swimming, such as stroke rate, velocity, etc. The sensors are mounted on the head of the participant for $100 \mathrm{~m}$ freestyle, breaststroke and butterfly swimming in $50 \mathrm{~m}$ swimming pool. There are 21 participants (12 males and 9 females with different ages) for evaluation. The accelerometer and GPS data are used to calculate the stroke count and the velocity. It is found that stroke count and velocity measures from accelerometer/GPS sensors are highly correlated to the measures from the reference video data. Therefore, this BSN system can be beneficial to the swimmers and trainers to find kinematic measures of performance such as velocity, acceleration, stroke rate, stroke length, stroke count, etc.

A detailed study for measuring kinematic variables in front crawl swimming using multiple accelerometers (attached to swimmer's wrist) is reported in Ref. ${ }^{[2]}$ The analysis has shown how lap time, velocity, stroke count, stroke duration, stroke rate, phases of the stroke are calculated and why they are promising to quantify the swimmers performance after validating with video-based results.

Using a single wearable IMU sensor placed on the sacrum, breaststroke swimming velocity is estimated in Ref. ${ }^{[23]}$ from velocity and acceleration data based on statistical (Bayesian) approach. Bayesian linear regression (BLR) is applied to estimate model parameters of a velocity model iteratively based on maximum posteriori (MAP) criterion and thereby, calculates the cycle mean velocity (CMV). The results are obtained for a total of 658 cycles collected from 15 participants (both well-trained and recreational) providing precise and more consistent results for different velocity-range among various participants compared to the results in Ref. ${ }^{[24]}$ obtained by Gaussian process regression (GPR) model, which is computationally more demanding.

Using a single IMU sensor (accelerometers) and an optical wireless transmitter (mounted on wrist) as well as optical wireless receiver (mounted on the goggles), a real-time BSN system is proposed in Ref. ${ }^{[25]}$ for generating swimming feedback to improve swimmers performance. A male recreational swimmer is asked to swim in a $50 \mathrm{~m}$ pool with different swimming styles including freestyle, backstroke, breaststroke and butterfly. From the raw acceleration ( $y$-axis) data, the maximums algorithm at the transmitter calculates the stroke rate by detecting the peaks. The stroke rate and the time difference between strokes are then sent to the receiver to activate a RGB LED and the swimmer is instructed to alert accordingly, i.e. red color for too slow stroke rate, green color for correct pace, and blue color for too fast stroke rate. The block diagram of the corresponding BSN system for swimming is shown in Figure 12.

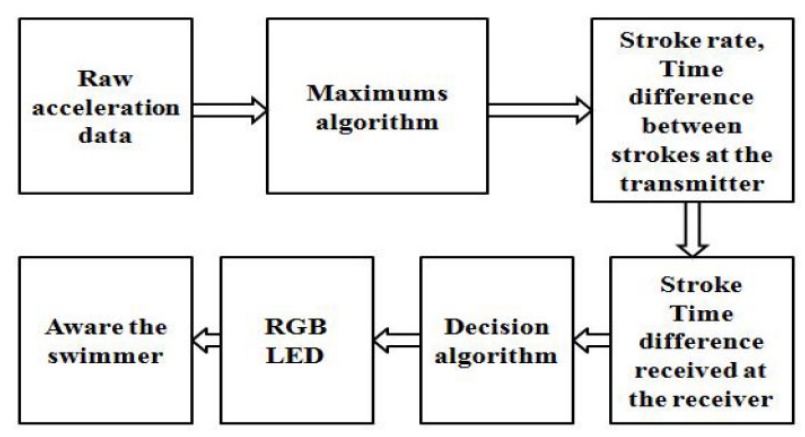

Figure 12. Transmitter and receiver's algorithm of the BSN used by swimmers

In Ref., ${ }^{[26]}$ a single IMU sensor, which is attached to the sacrum, is used to generate a lap velocity profile for freestyle swimming. The 3-axis acceleration data from 17 swimmers (8 junior and 9 retired persons) and 15 laps at three different efforts (5 laps comfortable, 5 laps training and 5 laps race pace) are used to derive swimmer's velocity profiles at different efforts and show a good match with the velocity profiles obtained from a tethered velocity meter.

A real-time BSN system for performance monitoring in swimming using a 3 axes accelerometer is presented in Ref. ${ }^{[27]}$ A wrist worn device is developed to estimate the performance parameters, such as stroke count, lap 
time/count/speed, total swimming time/distance, swimming efficiency in backstroke, breaststroke and front crawl swimming. Using the data from 13 different swimmers consists with 646 laps and 858.78 min duration, the iterative method gives high accuracies such as $>99 \%$ for precision/sensitivity for lap detection and $>92 \%$ for style classification.

The work in Ref. ${ }^{[28]}$ deals with automatic arm stroke phase detection for front-crawl swimming using three IMU sensors to replace video-based system for temporal phase detection during swimming. The three sensors are attached to the left arm, right arm, and sacrum. The timing of pull, push and recovery phases for both arms as well as index of coordination (IdC) parameter are tracked by Kalman filtering using 3D acceleration and 3D angular velocity data. The change of slope, orientation and peak information are used to track the different phases showing high accuracies.

In Ref., ${ }^{[29]} 3$-axial acceleration and 3-axial gyroscope data from wearable IMU sensors are used to track the phases of canoe stroke into propulsion phase and recovery phase for performance assessment in canoe. The sensors are attached to rower's body (upper arm, forearm, leg, chest, abdomen). A time-series segmentation algorithm is used for phase identification as follows: Firstly, three types of features are calculated by using normal distribution of the data. Secondly, the phase labels are predicted by training the features using a SVM classifier. Finally, phase reconstruction is performed for reducing the false predicted labels by extracting adaptive time threshold and magnitude threshold.

In Ref., ${ }^{[30]} 19$ different types of golf swing (straight, pull, push, slice, draw, hook, fade, etc.) are classified using accelerator, gyroscope and strain gage sensors (attached to golf club) based on various representative convolutional neural networks, i.e. deep convolutional neural networks (DCNN). The CNN architectures used to classify the golf swing data are Vanila CNN, VGG-like CNN, Inception-based CNN, Residual-block-based CNN are adopted and their implementational details are presented as well as the classification results are compared with the SVM classifier.

An IMMU (Inertial Magnetic Measurement Unit) sensor based landing momentum determination approach is presented in Ref. ${ }^{[31]}$ for ski jumping. The sensors are attached to both skies. The IMMU data is preprocessed followed by velocity calculation for the landing analysis. The IMMU based landing momentum is obtained by multiplying the athlete's mass with the vertical landing velocity. For testing, four ski jumpers are participated who jumped from an assigned hill, while three of the four jumping give less than $10 \%$ error when comparing the results with the force measurement reference system.
The study in Ref. ${ }^{[32]}$ analyses the motion between a horse and its ride based on inertial measurement system where the sensors are attached to rider's body (head, chest, pelvis, arms, thighs and calves). This motion varies due to the sitting position of a professional rider and beginner. A kinematic analysis of different riders' posture depending on the sitting position is done based on the BSNs. It can identify the level of skill of a rider and give feedback accordingly to an equestrian. The rider's poster is estimated by combining the gradient descent algorithm with a human biomechanical model to track the ride's motion. Analysing the exercise intensity of pelvis in two horse gaits (walking and rising trot), the difference between skill levels of the riders is distinguished.

In Ref., ${ }^{[33]}$ various badminton strokes are recognized based on BSN. The system contains wireless inertial sensor nodes, wireless receiving node and an operating system (PC). Each wireless inertial sensor node is comprised of an accelerometer and gyroscope attached to player's body (both wrists, waist, and right ankle). At first, the strokes are detected from the non-strokes motions using the preprocessed acceleration and gyroscope data sampled at $100 \mathrm{~Hz}$. Then a two-layer HMM (Hidden Markov Model) classifier is used to recognize 14 different strokes after feature extraction and feature selection from the segmented data obtained by the proposed window segmentation method named as WCSP (window with containing the stroke points). The classification accuracy is evaluated by considering all the possible sensor combinations. The block scheme of the corresponding BSN system for badminton is presented in Figure 13.

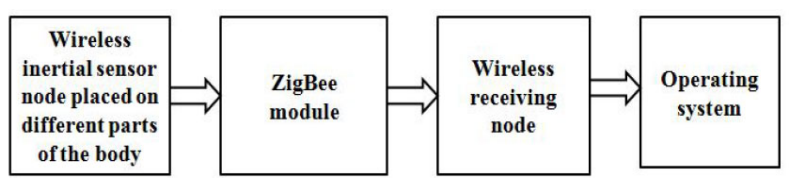

Figure 13. Overview of the system platform considered in badminton

5. TyPES OF SPORTS, WEARABLE SENSORS, MEASURED DATA, FEATURES/VARIABLES, DATA ANALYSIS, BASIC CLASSIFIER MODELS, AND THE RESULTS OF EVALUATION/PERFORMANCE OF THE SENSOR FACILITATED BY BSN AS WELL AS THE PROBLEMS RELATED TO THE STUDIES TOGETHER WITH SOME NOTES

We have tabulated in various types of sports facilitated by BSN, such as swimming, tennis, table tennis, ski jumping, ice hockey, skating, golf , badminton, horse riding, canoe as 
presented in Table 1. Most of the studies have been done for swimming.

Table 1. Types of sports facilitated by BSN and the corresponding references

\begin{tabular}{c|c}
\hline \hline Sports & References \\
\hline \hline Swimming & {$[13],[17],[21],[22],[23],[24],[25],[26],[27],[28]$} \\
\hline Ski jumping & {$[18],[31]$} \\
\hline Ice hockey & {$[14]$} \\
\hline Skating & {$[14]$} \\
\hline Golf & {$[16],[30]$} \\
\hline Table tennis & {$[20]$} \\
\hline Tennis & {$[12]$} \\
\hline Snowboarding & {$[19]$} \\
\hline Canoe & {$[29]$} \\
\hline Horse riding (Equestrian sports) & {$[32]$} \\
\hline Badminton & {$[33]$} \\
\hline
\end{tabular}

Table 2 presents a list of wearable sensors as well as the measured data to monitor the performance in various sports where all studies are facilitated by the accelerometer.

Table 2. Types of sensors used for BSN systems in performance analysis for various sports and the corresponding references

\begin{tabular}{|c|c|c|}
\hline Reference & Sensors & Measured data \\
\hline$[13]$ & Accelorometer, Gyroscope & 3-axial accelerations and angular velocities \\
\hline$[17]$ & Accelorometer & 3-axial acceleration data \\
\hline [21] & Accelorometer, GPS & GPS and 3-axial acceleration data \\
\hline [22] & Accelorometer & 3-axial acceleration data \\
\hline [23] & Accelorometer, Gyroscope & Swimming inertial data \\
\hline$[24]$ & Accelorometer, Gyroscope & Swimming inertial data \\
\hline [25] & Accelorometer & Acceleration data \\
\hline$[26]$ & Accelorometer & 3-axial acceleration data \\
\hline$[27]$ & Accelorometer & 3-axial acceleration data \\
\hline$[28]$ & Accelorometer, Gyroscope & Swimming inertial data \\
\hline$[18]$ & Accelorometer, Gyroscope & Jump data in ski jumping \\
\hline$[14]$ & Accelorometer, Gyroscope & Motion data of the players \\
\hline [16] & Accelorometer, Gyroscope & 3-axial accelerations and angular velocities \\
\hline [20] & Accelorometer, Gyroscope & Basic table tennis stroke data \\
\hline$[12]$ & $\begin{array}{c}\text { Accelorometer, Gyroscope, } \\
\text { Magnetometer }\end{array}$ & $\begin{array}{c}\text { Locomotion and } \\
\text { tennis shot movement data }\end{array}$ \\
\hline [19] & $\begin{array}{c}\text { Accelorometer, Gyroscope, } \\
\text { Magnetometer }\end{array}$ & $\begin{array}{l}\text { Jumper's trick events } \\
\text { (Grind and Air) data } \\
\end{array}$ \\
\hline [29] & Accelorometer, Gyroscope & Player's motion data \\
\hline$[30]$ & $\begin{array}{l}\text { Accelorometer, Gyroscope, } \\
\text { and Strain gage }\end{array}$ & $\begin{array}{c}\text { Golf swing data } \\
\text { and golf club shaft bend data }\end{array}$ \\
\hline$[31]$ & $\begin{array}{c}\text { Accelorometer, Gyroscope, } \\
\text { Magnetometer, Temperature sensor }\end{array}$ & $\begin{array}{l}\text { Sky jump data } \\
\text { and temperature }\end{array}$ \\
\hline$[32]$ & $\begin{array}{c}\text { Accelorometer, Gyroscope, } \\
\text { Magnetometer }\end{array}$ & $\begin{array}{l}\text { Rider's attitude } \\
\text { and motion data }\end{array}$ \\
\hline$[33]$ & Accelorometer, Gyroscope & Badminton motion data \\
\hline
\end{tabular}

Several papers have investigated performance monitoring in various sports using different classifiers as shown in Table 3. However, most of the papers mainly focus on the use of Support Vector Machine (SVM) classifier providing higher performance than most of the other classifiers used.

In Table 4, the performances of the sensors or the results of evaluation are presented for the references not involved with classification as shown in Table 3. Various types of accuracies including different statistical errors, correlation, significance test, etc. are used in different studies as shown by Table 4 .

Table 3. Types of classifiers used as well as the classification results/performance of the sensor in performance monitoring for various sports and the corresponding references

\begin{tabular}{|c|c|c|c|c|}
\hline Reference & Classifier & Contribution & $\begin{array}{l}\text { Classification } \\
\text { Accuracy }\end{array}$ & Subjects \\
\hline [14] & Random Forest & $\begin{array}{l}\text { Recognize eight skating } \\
\text { motions in skating }\end{array}$ & $92 \%$ & 19 (11 amateur \\
\hline$[14]$ & $\begin{array}{l}\text { Support Vector } \\
\text { Machine (SVM) }\end{array}$ & $\begin{array}{l}\text { Recognize a shot to } \\
\text { assess a player's skill } \\
\text { level in ice hockey }\end{array}$ & $92.9 \%$ & \& 8 professional) \\
\hline [20] & SVM & $\begin{array}{l}\text { Classify basic stroke } \\
\text { type in table tennis }\end{array}$ & $96.7 \%$ & 10 \\
\hline$[12]$ & \begin{tabular}{|c|} 
Longest Common \\
Subsequent (LCSS)
\end{tabular} & $\begin{array}{l}\text { For shot classification } \\
\text { in tennis }\end{array}$ & $94 \%$ & 4 \\
\hline [12] & SVM & $\begin{array}{c}\text { For step classification } \\
\text { in tennis }\end{array}$ & $95 \%$ & \\
\hline [19] & SVM & $\begin{array}{l}\text { For grind classification } \\
\text { in snowboarding }\end{array}$ & $90.3 \%$ & 4 \\
\hline [19] & $\mathrm{kNN}$ & $\begin{array}{l}\text { For air classification } \\
\text { in snowboardng }\end{array}$ & $93.3 \%$ & \\
\hline [29] & SVM & $\begin{array}{c}\text { For motion phase classification } \\
\text { in canoe }\end{array}$ & Not described & 2 \\
\hline [30] & $\begin{array}{c}\text { Deep Convolutional } \\
\text { Neural Networks } \\
\text { (DCNNs) }\end{array}$ & $\begin{array}{l}\text { For swing } \\
\text { classification } \\
\text { in golf }\end{array}$ & $\begin{array}{c}\text { Vanila CNN: } 95 \%, \\
\text { VGG CNN: } 97 \% \text {, } \\
\text { Inception CNN: } 97 \% \\
\text { ResNet CNN: } 92 \%\end{array}$ & 4 \\
\hline [33] & HMM(Layer two) & $\begin{array}{l}\text { For stroke recognition } \\
\text { in badminton }\end{array}$ & $97.96 \%$ & 12 \\
\hline
\end{tabular}

Table 4. The results of evaluation/performances of the sensors for the references other than in Table 3 (RMSE: Root-mean square error, $r$ : Correlation coefficient, $p$ : Significance, MAE: Maximum absolute error, CMV: Cycle mean velocity, $d$ : Input space dimension for Bayesian regression estimation, ICC: Intra-class correlation)

\begin{tabular}{|c|c|c|}
\hline Reference & Performance/Results of Evaluation & Subjests \\
\hline [13] & Not described & 1 \\
\hline$[16]$ & Not described & 1 \\
\hline$[17]$ & $\begin{array}{c}\text { Session time improvement: } 10.1 \% \text { (Max.); } \\
\text { Swimmer's rotation improvement:18.5\% (Max.) }\end{array}$ & 4 \\
\hline$[18]$ & $\begin{array}{l}\text { Orientation angle(left ski) RMSE: } 9.3 \& \text { std dev.: } 1.8 \\
\text { Orientation angle(right ski) RMSE: } 2.0 \text { \& std dev.: } 2.0\end{array}$ & 3 \\
\hline [21] & $\begin{array}{l}\text { High correlation (between } 0.98 \text { and } 1 \text { ) } \\
\text { for stroke count detection }\end{array}$ & $\begin{array}{l}21 \text { (12 male } \\
\& 9 \text { female) }\end{array}$ \\
\hline$[22]$ & $\begin{array}{c}\mathrm{r}=0.97, p=0.00 \text { for lap times } \\
\mathrm{r}=0.94, p=0.00 \text { for stroke count } \\
\mathrm{r}=0.92, p=0.00 \text { for stroke rate } \\
\mathrm{r}=0.63, p=0.00 \text { for stroke duration } \\
\text { MAE: } 0.06 \mathrm{~s}, 0.07 \mathrm{~s}, 0.06 \mathrm{~s}, 0.08 \mathrm{~s} \\
\text { for phase durations (Entry, Pull, Push, and Recovery) }\end{array}$ & 12 \\
\hline$[23]$ & $\begin{array}{c}\text { CMV error }=0.0 \pm 13.6(d=5) \\
\text { CMV error }=0.3 \pm 11.1(d=7) \\
\text { CMV error }=0.1 \pm 9.6(d=9)\end{array}$ & 15 \\
\hline$[24]$ & CMV error $=-0.7 \pm 6.8$ & $\begin{array}{l}20 \text { (13 male } \\
\& 7 \text { female) }\end{array}$ \\
\hline [25] & Percentage of highest error-free received data $=60.3 \%$ & 1 \\
\hline$[26]$ & $\begin{array}{c}\text { Lap velocity distribution errors: mean skewness }=0.96 \pm 0.47 \\
\text { mean kurtosis }=2.93 \pm 1.12\end{array}$ & 17 \\
\hline$[27]$ & Average relative error $<1.2 \%$ for stroke count & 13 \\
\hline$[28]$ & $\operatorname{ICC}(1,1)=0.94$ & $\begin{array}{c}7(5 \text { male } \\
\& 2 \text { female })\end{array}$ \\
\hline$[31]$ & Average momenta accuracy $=85 \%$ & 1 \\
\hline$[32]$ & $\begin{array}{c}\mathrm{r}=0.61,0.94 \text {, and } 0.51 \text { for } 3 \text { joint angles in walking } \\
\mathrm{r}=0.14,0.12 \text {, and } 0.11 \text { for } 3 \text { joint angles in rising trot }\end{array}$ & $\begin{array}{l}10 \text { ( } 5 \text { professional } \\
\text { riders \& } 5 \text { beginners })\end{array}$ \\
\hline
\end{tabular}


Table 5. Types of features/variables, data analysis used for BSN systems in performance monitoring for various sports and the corresponding references

\begin{tabular}{|c|c|c|}
\hline Refer & Features & Data Analysis \\
\hline [13] & $\begin{array}{l}\text { Stroke type, Lap time, Lap count, } \\
\text { stroke frequency and Distance per stroke }\end{array}$ & $\begin{array}{l}\text { Moving Average filtering of acceleration and } \\
\text { gyroscope data to find breathing pattern }\end{array}$ \\
\hline [17] & Lap time, Rotation status, Stroke rate & Raw acceleration data \\
\hline$[21]$ & $\begin{array}{l}\text { Stroke counts for front-crawl, } \\
\text { breaststroke, backstroke }\end{array}$ & $\begin{array}{l}\text { Raw acceleration data; Calculate Pearson's correlation coefficient } \\
\text { (correlation between } 0.98 \text { and } 1 \text { ) }\end{array}$ \\
\hline [22] & $\begin{array}{l}\text { Lap time, Velocity, Stroke count } \\
\text { Stroke duration, Stroke rate, Stroke phases }\end{array}$ & $\begin{array}{l}\text { Calculate Kolmogorov-Smirnov test, } t \text {-Test, Pearson's correlation coefficient, } \\
\text { Different types of errors }\end{array}$ \\
\hline [23] & Breast stroke velocity & $\begin{array}{c}\text { Bayesian algorithm, estimate velocity model parameters iteratively using } \\
\text { maximum posteriori (MAP) criterion and calculate cycle mean velocity (CMV) }\end{array}$ \\
\hline [24] & Front crawl velocity & $\begin{array}{c}\text { Gaussian process (GP) regression framework or parameter learning } \\
\text { to calculate cycle mean velocity (CMV) }\end{array}$ \\
\hline [25] & Stroke rate & Maximums algorithm to calculate stroke rate and the time difference between strokes \\
\hline [26] & $\begin{array}{c}\text { Stroke rate } \\
\text { Mean velocity }\end{array}$ & $\begin{array}{l}\text { Lowpass filtering of raw acceleration data to find highpass data; Total acceleration } \\
\text { data by summing the data in } x, y, z \text { axes; Velocity profile by integrating } \\
\text { total acceleration data (using trapezoidal rule) over time }\end{array}$ \\
\hline [27] & $\begin{array}{l}\text { Lap count, Stroke count, Lap time, } \\
\text { Total swimming time, Swimming efficiency }\end{array}$ & $\begin{array}{l}\text { Iterative algorithms in an ample-by-sample basis, Speed per lap, Total swam distance, } \\
\text { State-machine and spectral analysis, Decision graph of depth two }\end{array}$ \\
\hline [28] & $\begin{array}{c}\text { Stroke phases } \\
\text { Inter-arm coordination }\end{array}$ & $\begin{array}{c}\text { Temporal phase detection algorithm based on slope tracker by Kalman filtering, adaptive } \\
\text { slope change detection using CUSUM, orientation estimation and peak detection }\end{array}$ \\
\hline [18] & $\begin{array}{l}\text { 3D orientation of left and right skies, } \\
\text { Angle of attack }\end{array}$ & $\begin{array}{c}\text { Initial sensor calibration; Initial rest vector, gravity vector alignment } \\
\text { Descent trajectory, rotation vector alignment; Quaternion-based } \\
\text { integration of gyroscope data }\end{array}$ \\
\hline [14] & $\begin{array}{c}\text { Power strokes, Breaking, Turns, Jumps } \\
\text { and Curves for skating } \\
\text { Slap and wrist shots for shooting in ice hockey }\end{array}$ & $\begin{array}{l}\text { Feature extraction and template matching for skating action detection, } \\
\text { Warping LCSS algorithm for classification of staking action } \\
\text { Feature extraction (maximum, minimum, mean rotational velocities) } \\
\text { followed by recognition of shoot types using Random Forest classifier }\end{array}$ \\
\hline [16] & Backswing, Downswing, Impact, Follow-through & Raw acceleration and gyroscope data \\
\hline [20] & $\begin{array}{l}\text { drive, push, block, and topspin with forehand } \\
\text { drive, push, block, and topspin with backhand }\end{array}$ & $\begin{array}{l}\text { Event detection algorithm based on energy calculation, highpass filtering, } \\
\text { negative masking and peak detection for stroke detection; SVM with } \\
\text { RBF kernel provides the best result for stroke type classification }\end{array}$ \\
\hline [12] & $\begin{array}{c}\text { Forehand and Backhand with Topspin and Slice, } \\
\text { Smash, Footsteps (Shot steps and Side steps) }\end{array}$ & $\begin{array}{l}K \text {-means clustering, Similarity measure and non-maximum suppression } \\
\text { for segment detection and LCSS classifier for shot classification; } \\
\qquad \mathrm{SVM} \text { classifier for step recognition }\end{array}$ \\
\hline [19] & Grind tricks, Air tricks & $\begin{array}{l}\text { Preprocessing, threshold analysis for trick event detection, and } \\
\text { classification of trick category using different types of classifiers }\end{array}$ \\
\hline [29] & Canoe sprint phases & $\begin{array}{c}\text { Time series segmentation, feature extraction for describing motion } \\
\text { phases, and SVM classification for motion phase }\end{array}$ \\
\hline [30] & $\begin{array}{l}\text { Different types of golf swing(straight, pull, push, } \\
\text { slice, draw, hook, fade, push-slice, pull-hook, etc. }\end{array}$ & $\begin{array}{l}\text { Preprocessing by data augmentation, data shuffling, and data } \\
\text { standaridization followed by classification with customized deep CNNs }\end{array}$ \\
\hline [31] & Landing momentum & $\begin{array}{l}\text { Preprocessing the data by a sensor calibration and alignment; Landing velocity } \\
\text { calculation to measure the momentum of landing phase }\end{array}$ \\
\hline [32] & $\begin{array}{l}\text { Horse gaits (Walking, Rising trot) } \\
\text { for horse riding (Equestrian sports) }\end{array}$ & $\begin{array}{l}\text { Sensor error calibration; Calibration of equestrian poster based on A-POS; } \\
\text { Quaternion update based on gradient descent algorithm; Equestrian motion } \\
\text { tracking based on quaternion; Analysis of riding poster based on Equestrian }\end{array}$ \\
\hline [33] & $\begin{array}{l}\text { Different strokes (serve, clear, push, } \\
\text { lob, chop, rushing, hook for fore- } \\
\text { hand \& backhand) in badminton }\end{array}$ & $\begin{array}{l}\text { Sample set generation by wavelet denoising, badminton stroke detection, window } \\
\text { segmentation, feature extraction and selection; classification by a two layer HMM classifier } \\
\text { (Layer 1: verify if the activity is a stroke, Layer 2: multiclass classification of the strokes) }\end{array}$ \\
\hline
\end{tabular}


In Table 5, the features/variables used in various sports together with the data analysis are summarized. As we see, the data analysis are mainly involved with signal processing (e.g. denoising, segmentation, detection, estimation, data alignment), pattern recognition including feature extraction, feature selection, and machine learning algorithms.

In Table 6, sampling rates used in sports performance monitoring are demonstrated, where it can be seen that the sampling frequency of $100 \mathrm{~Hz}$ is mostly used for the wearable sensors.

Table 6. Sampling rate used in sports performance monitor studies

\begin{tabular}{c|c|c|c|c|c}
\hline \hline Sampling rate (Hz) & 10 & 50 & 100 & 200 & 1000 \\
\hline References & {$[17,27]$} & {$[16]$} & {$[13,14,18,21,29,32,33]$} & {$[12,18]$} & {$[20,30]$} \\
\hline \hline
\end{tabular}

In Table 7, the problems associated with various studies together with some corresponding notes are presented. As we see, various issues like data transmission, sensors positions, sensors fusion, complexity, flexibility, etc. can be drawn into our attention.

\section{General remarks}

From Table 2, it can be seen that only few studies have used three sensors (accelerometer, gyroscope, and magnetometer), i.e. IMU-unit. The use of the three sensors together could provide improvements of the BSN for sports performance. Based on the results in Table 3, the works involved with limited number of subjects do not give high classification accuracy and vice versa. So, the number of participants (including male, female, professional and amateur players) should be sufficient, or the data size needs to be large enough to achieve better performance by any BSN system. As it can be seen in Table 4, the type error used is either RMSE or MAE for performance evaluation. It would be preferable to consider different types of errors or at least more than one type error for more convincing performance evaluation. From Table 5, it can be seen the work like in, ${ }^{[32]}$ for example, has shown the body movement representation or kinematic analysis. However, it should be interesting to incorporate this type of analysis to make the study more intuitive. Also, one BSN system is dedicated to only one type of sport except the work in, ${ }^{[14]}$ which can be used for multiple sports, i.e. both for ice hockey and skating. This versatility of a BSN system can be an important consideration in method development. From Table 7, it is found that all studies have not addressed the computational complexity even the detailed papers with no other problems have missed only the computational complexity (e.g. $\mathrm{in}^{[33]}$ ). This is an important issue to take into account for real-time implementation of a BSN system besides its performance. Also, some studies are involved with wireless communication need to be more efficient and secure with less error free data transfer (e.g. in ${ }^{[13,25]}$ ). In such case, Bluetooth (BT) technology can be used for wireless data transmission, or a cloud assisted BSN system can be proposed. The optimization in terms of sensors positions is another important issue, which is missing in all studies. Then a method or a system can be generalized in terms of applications, for example a BSN system can be used for all types of swimming styles (i.e. free-crawl, backstroke, breaststroke and butterfly), unlike some methods (e.g. ${ }^{[24,28]}$ ). Moreover, some systems are useful for either indoor or outdoor games (e.g. in ${ }^{[21]}$ ). The systems need to be designed so that they are useful for both types of competitions. Further, the studies can improve their performance by sensors fusion like the one demonstrated in. ${ }^{[33]}$

\section{VIDEO CAMERA BASED SYSTEMS USED IN VARIOUS SPORTS FOR ILLUSTRATIVE COMPARISON}

In, ${ }^{[34]}$ a study is done to monitor golf-swing performance using 8 video cameras. Three different swing phases, i.e. backswing, downswing, and follow-through, are measured and cross-correlation analysis of time-series signals are performed between joints and the club during the full golf swing using a 3D motion capture system. The quality of the forehand smash in badminton is measured in ${ }^{[35]}$ using a video camera based system consists of 10 cameras. The trunk rotation (X-Factor) is shown to be an important parameter which is found to be vital to maximize the release speed of the shuttlecock. In, ${ }^{[36]}$ the rowers' movements is captured using an optical system using 8 video cameras. The rowers performance is monitored on the ergometer over a distance of $500 \mathrm{~m}$ by measuring time(s)/100 m, time(s)/500 m, number of drags (strokes) per min, and power (W). The work in $^{[37]}$ estimates the stroke rates of the swimmers by detecting the swimmers and their poses using video data in the swimming pool. The approach is evaluated at a wide variety of swimmers including male/female and various age group with different swimming speeds using nine videos with the lengths vary from 20 to 60 seconds and are recorded at 50 frames per second.

An investigation has been made on the precision and reliability using a new optical player tracking system to determine the displacement of the football players. The participant's positions in the field have been determined on a $105 \mathrm{~m} \times 68$ $m$ playing area using eight immobile high-definition video cameras (Legria HF R38, Canon, Tokyo, Japan). Moreover, two video cameras consisting $20 \mathrm{~m}$ of height have been setup in four corners of the field and that permit continuous and clear vision of the area. ${ }^{[38]}$ 
Table 7. Types of problems associated with notes and the corresponding references

\begin{tabular}{|c|c|c|}
\hline Reference & Problems & Notes \\
\hline$[13]$ & Wireless communication is off line and not robust. & $\begin{array}{c}\text { Bluetooth (BT) can be used for wireless data } \\
\text { transmission due to its pervasiveness and low-power } \\
\text { consumption in real-time wearable system. }\end{array}$ \\
\hline$[17]$ & $\begin{array}{l}\text { Not sufficient accuracy for feedback } \\
\text { information to the swimmers. }\end{array}$ & $\begin{array}{c}\text { Needs more participants, more accurate } \\
\text { time keeping as well as more appropriate } \\
\text { swimming techniques for real-time feedback. }\end{array}$ \\
\hline [21] & $\begin{array}{l}\text { Stroke count in free style swimming using head mounted } \\
\text { accelerometer is not accurate. }\end{array}$ & $\begin{array}{l}\text { Integrated accelerometer and GPS technology } \\
\text { is not suitable not for indoor swimming. }\end{array}$ \\
\hline$[22]$ & $\begin{array}{l}\text { Single sensor system (e.g. sacrum mounted) is not able to } \\
\text { determine or infer the phases of the stroke with } \\
\text { sufficient accuracy. }\end{array}$ & Needs to incorporate multiple sensor systems. \\
\hline [23] & $\begin{array}{l}\text { Wireless data transfer and real-time implementation of the } \\
\text { system need to be considered. }\end{array}$ & $\begin{array}{l}\text { The real-time monitoring of the velocity can be useful } \\
\text { for swimming pattern anomaly detection that is } \\
\text { very important to improve open-water swimming safety. }\end{array}$ \\
\hline [24] & $\begin{array}{l}\text { Useful only for front-crawl swimming in } \\
\text { cycle mean velocity (CMV) estimation. }\end{array}$ & $\begin{array}{l}\text { Unlike camera-based system or tethered device, } \\
\text { the system does not have limited capture volume which } \\
\text { is suitable to use in open water. }\end{array}$ \\
\hline$[25]$ & The highest error free received data is only $60.3 \%$. & The experimental results are limited. \\
\hline [26] & The method works well for only freestyle swimming. & The results depend on the swimming style of the swimmer. \\
\hline [27] & The system does not work for butterfly swimming style. & $\begin{array}{l}\text { Providing high accuracy for the swimming activities in } \\
\text { backstroke, breaststroke and front crawl swimming styles. }\end{array}$ \\
\hline$[28]$ & $\begin{array}{l}\text { Applicable in temporal phase detection } \\
\text { only for front crawl swimming. }\end{array}$ & $\begin{array}{l}\text { Needs to show also the applicability } \\
\text { for other swimming styles. }\end{array}$ \\
\hline$[18]$ & Results are evaluated using RMS only. & $\begin{array}{l}\text { The method is simplified as it does not require additional } \\
\text { calibration. However, further improvement can be achieved } \\
\text { by increasing the angle determination accuracy. }\end{array}$ \\
\hline [14] & $\begin{array}{l}\text { The performance of the prototype gameplay } \\
\text { tracking system is not high giving only } 70 \% \\
\text { accuracy during ice hockey game. }\end{array}$ & The system can be used for both ice hockey and skating. \\
\hline [16] & The method is not automatic. & Preliminary results are presented. \\
\hline$[20]$ & The system does not have real-time ability. & $\begin{array}{c}\text { Provide high recognition accuracy and has potential to } \\
\text { use the system as a training device. }\end{array}$ \\
\hline [12] & More training data is required. & $\begin{array}{l}\text { The system can automatically give recommendations } \\
\text { on the timing of the tennis player. }\end{array}$ \\
\hline [19] & $\begin{array}{l}\text { Preliminary studies are done in tricks classification for } \\
\text { freestyle snowboarding. }\end{array}$ & $\begin{array}{l}\text { The method is promising and needs to be implemented } \\
\text { in a real-time capable system. }\end{array}$ \\
\hline [29] & Only a single classifier (SVM) is used. & $\begin{array}{l}\text { Cloud assisted BSN approach can be exploited to solve } \\
\text { real-time remote data transmission to provide } \\
\text { an on-line motion monitoring system. }\end{array}$ \\
\hline [30] & The computational complexity is not addressed. & $\begin{array}{l}\text { Representative Deep CNN-based advanced } \\
\text { classifiers are used. }\end{array}$ \\
\hline [31] & Limited subjects are considered. & $\begin{array}{l}\text { The method leads to be an unobtrusive and low-cost ski } \\
\text { landing analysis system. However, more subjects are } \\
\text { to be considered for better results. }\end{array}$ \\
\hline [32] & $\begin{array}{l}\text { Comparative such as Kalman filter based tracking } \\
\text { results are not presented. }\end{array}$ & $\begin{array}{l}\text { Multiple sensors are used and detailed statistical analysis } \\
\text { is presented. Most importantly body movement (kinematic) } \\
\text { analysis is shown based on quaternion. }\end{array}$ \\
\hline $\begin{array}{l}{[33]} \\
\end{array}$ & $\begin{array}{l}\text { Complexities of the proposed window } \\
\text { segmentation method is not compared. }\end{array}$ & Detailed results by sensors fusion are presented. \\
\hline
\end{tabular}


Subsequently, wearable sensors have also been utilized to determine the corroboration of the bowling action, both for arm flex measurement and release point detection in cricket. It can help both the players and the match officials to find out if the bowlers are bowling within the guidelines whereas the setup cost is very cheap. However, to determine the faults or checking bowling actions, a motion analysis system is needed which should contain at least 12 high speed cameras, causing the arrangements highly expensive. ${ }^{[39]}$

From the above illustration, it is found that a typical video based system would consist of as many as 8 cameras for which the system is expensive and the set up as well as processing are time-consuming.

\section{CONCLUSION AND OPEN ISSUES}

In this paper, we have overviewed the recent progress which have been made on BSN for performance analysis in sports. Various sports were considered and a compatibility between sports, sensors, features, data analysis and classifiers as well as their results of evaluation/performance is shown. The BSN systems with inertial sensors are replacing the video-based systems for performance monitoring in sports and removes considerably the burden of demanding camera installation and calibration procedure as well as the time consuming video processing. One key feature is to design a BSN system as an embedded system with less memory and computational power to develop a small wearable device. Thereby, competition matches can be more efficiently analyzed, while amateur players can use it as a training device to improve their own skills and share their performances with other professional players. However, most of the used sensors record the data in a memory and then post processing can be applied. Therefore, it requires to provide real-time solutions for the existing BSN systems in sports.

One new issue is to replace Umpire Decision Review System (UDRS or DRS) in the sport of cricket $^{[40]}$ by proposing a BSN system for decision making during the match. The current DRS is a video-based system which requires expensive installation or setup cost and also time consuming to make a decision by the third Umpire. The BSN system, on the other hand, can be much less expensive and fast. More importantly, it could provide more correct decisions than the current video-based DRS due to multi-modal motion analysis of the incoming ball using 3D acceleration and 3D rotation data from multiple wearable inertial sensors. Thereby, an automatic and instant feedback can be sent to the Umpire about the decision (e.g. leg-before-wicket [LBW], caught behind, etc.). The decision of LBW can be made by using the wearable sensors in the pads. The 3D trajectory of the cricket ball can be calculated by 1D Kalman filtering ${ }^{[41]}$ of each acceleration data in the $x-y-z$ axis and check whether the ball would hit the wicket(s) after hitting the pad in front of the wickets. If yes, the Umpire will confirm the decision for LBW. The decision of caught behind can be facilitated by the wearable sensors in the cricket bat. The accelerations in the $x-y-z$ axis can be measured using a 3D accelerometer to find out whether there is a sudden increase in the acceleration values. If it happens, the Umpire will confirm that the ball has touched the bat as well as the caught behind decision.

The context-aware BSNs can be another future insight to handle diverse dataset due to different positions of the sensors. In this case, the position of a body sensor attached to may be an important property in the sensor profile. ${ }^{[42]}$ If the dataset is not annotated (or data labels are unavailable), a method to encode the background knowledge, like the sensor profiles, is required. Such design can facilitate each sensor in the source-domain dataset to match its counterpart in the target domain. In such case, the IMU consisting of accelerometer, gyroscope, magnetometer together with a position sensor providing the contextual information about the position of the body sensor attached, can be used together. The other future aspect is to look for collaborative $\mathrm{BSNs}^{[42]}$ where different BSNs able to collaborate with each other to fulfill a common goal. For example, under multisensor data fusion schema, a BSN system monitoring sports performance and a BSN system capturing health/safety attribute (e.g. dehydration,stress level) can be fused. These context-aware and collaborative BSNs can be useful for long-term performance monitoring using big sports data. The measured data of the multiple sensors from the two BSN systems can be weighted using the estimation equations in $^{[43]}$ for data fusion. The output would be then used to monitor a more sophisticated sports performance for a span of time. For example, the stroke rate calculated from peaks of the acceleration data and the heart-rate variability from angular velocity of the gyroscope data can be fused for long-term monitoring of the swimmer's performance at different stress level over a period of time.

Another open issue is to explore BNS systems for predicting future performance in sports referring to an initial study in. ${ }^{[4]]}$ The performance rating for every player of the current game is predicted in $^{[44]}$ using individual models of every player, average model adapt to the mean performance of all players, the performance of every player in previous games. Multitask ridge regression and multitask support vector regression (SVR) are proposed for learning the model parameters by minimizing the objective function (i.e. mean absolute error) to predict the future performance rating of the soccer players. The proposed multitask generalizations of ridge regression and support vector regression provide 
efficiently learning player-specific models. Many informative features (e.g. players identity, features from preceding games, such as goals, passes, dribbles, offense, defense, etc.) and feature selection using a modified recursive feature elimination strategy are used for optimization. A player's number of goals and dribbles, for instance, correlates positively with good grades, whereas the number of opponent shots correlates negatively with player scores. By predicting the ratings of every selected candidates, it could assist the main coach, main selectors, and captain in sports like cricket or soccer to select the final 11 players before the game.

\section{ACKNOWLEDGeMents}

The authors gratefully acknowledge the anonymous Reviewers and Associate Editor who enhanced this paper through their constructive feedback.

\section{REFERENCES}

[1] Poon CCY, Lo BPL, Yuce MR, et al. Body sensor networks: In the era of big data and beyond. IEEE Reviews In Biomedical Eng. 2015; 8: 4-16. PMid:25935046. https ://doi.org/10.1109/RB ME. 2015. 2427254

[2] Callaway AJ, Cobb JE, Jones I. A comparison of video and acceleation based approaches applied to performance monitoring in swimming. Int. Journal of Sports Science and Coaching. 2009; 4(9): 139-153. https ://doi .org/10.1260/1747-9541.4.1.139

[3] De Boer D, van Rheenen OS, van Zelm E, et al. Design considerations for a wearable sensor network that measures accelerations during Water-Ski jumping. IEEE Int. Conf. on Body Sensor Networks. 2013: $1-5$.

[4] Barshan B, Yüksek MC. Recognizing daily and sports activities in two open source machine learning environments using bodyworn sensor units. The Computer Journal. 2014; 57(11): 1649-1667. https ://doi.org/10.1093/comjnl/bxt075

[5] Cornacchia M, Ozcan K, Zheng Y, et al. A survey on activity detection and classification using wearable sensors. IEEE Sensors. 2017; 17(2): 386-402. https ://doi.org/10.1109/JSEN . 2016. 2628 346

[6] Seshadri DR, Drummond C, Craker J, et al. New integrated technologies allow coaches, physicians, and trainers to better understand the physical demands of athletes in real time. IEEE Pulse. 2017: 38-43.

[7] de Magalhaesa FA, Vannozzib G, Gattac G, et al. Wearable inertial sensors in swimming motion analysis: a systematic review. Journal of Sports Sciences. 2015; 33(7): 732-745. PMid:25356682. https ://doi.org/10.1080/02640414.2014.962574

[8] Guignard B, Rouard A, Chollet D, et al. Behavioral dynamics in swimming: the appropriate use of inertial measurement units. Frontiers in Psychology. 2017: 1-16.

[9] Lai X, Liu Q, Wei X, et al. A Survey of body sensor networks. Sensors. 2013; 13: 5406-5447. PMid:23615581. https://doi.org/ $10.3390 / \mathrm{s} 130505406$

[10] Zhang Z, Pansiot J, Lo B, et al. Human back movement analysis using BSN. Int. Conf. on Body Sensor Networks. 2011: 13-18.

[11] HuS S. Body sensor network for in-home personal healthcare. Doctor of Philosophy in Computer Engineering (PhD), Michigan Technological University. 2012. Available from: https://digitalcommons .mtu.edu/etds/63/

[12] Buthe L, Blanke U, Capkevics H, et al. A wearable sensing system for timing analysis in tennis. IEEE Int. Conf. Wearable and Implantable Body Sensor Networks (BSN). 2016: 43-48.

[13] Lecouterea J, Puersa R. Wireless communication with miniaturized sensor devices in swimming. Int. Conf. Sports Engineering Association. 2014 Procedia Engineering (Elsevier). 2014; 72: 398-403.
[14] Hardegger M, Ledergerber B, Mutter S, et al. Sensor technology for ice hockey and skating. IEEE Int. Conf. Body Sensor Networks. 2015: 1-6.

[15] Available from: https://en.wikipedia.org/wiki/F1\$_\$sco re\$\#\$Formulation

[16] Mitsui T, Tang S, Obana S. Support system for improving golf swing by using wearable sensors. Int. Conf. Mobile Computing and Ubiquitous Networking (ICMU). 2015: 100-101.

[17] Li R, Cai Z, Lee W, et al. A wearable biofeedback control system based body area network for freestyle swimming. IEEE Int. Conf. Eng. Med. \& Bio. Soc. (EMBC). 2016: 1866-1869.

[18] Groh BH, Weeger N, Warschun F, et al. Simplified orientation determination in ski jumping using inertial sensor data. IEEE Conf. Inertial Sensors and Systems. 2014: 11.

[19] Groh BH, Fleckenstein M, Eskofier BM. Wearable trick classification in freestyle snowboarding. IEEE Int. Conf. Wearable and Implantable Body Sensor Networks (BSN). 2016: 89-93.

[20] Blank P, Ho $\beta$ bach J, Schuldhaus D, et al. Sensor-based stroke detection and stroke type classification in table tennis. Int. Conf. Semantic Web (ICSW). 2015: 93-100.

[21] Beanlanda E, Maina LC, Aisbetta B, et al. Validation of GPS and accelerometer technology in swimming. Journal of Science and Medicine in Sport (Elsevier). 2014; 17: 234-238. PMid:23707140. https://doi.org/10.1016/j.jsams.2013.04.007

[22] Callaway AJ. Measuring kinematic variables in front crawl swimming using accelerometers: A validation study. Sensors. 2015; 15: 11363-11386. https://doi.org/10.3390/s150511363

[23] Dadashi F, Millet GP, Aminiana K. A Bayesian approach for pervasive estimation of breaststroke velocity using a wearable IMU. Pervasive and Mobile Computing (Elsevier). 2015; 19: 37-46. https://doi.org/10.1016/j.pmcj.2014.03.001

[24] Dadashi F, Millet GP, Aminiana K. Gaussian process framework for parvasive estimation of swimming velocity with body-worn IMU. Electronics Letters. 2013; 49(1): 44-46. https : //doi .org/10.1 049/el.2012.3684

[25] Hagema RM, Haelsig T, O'keefe SG, et al. Second generation swimming feedback device using a wearable data processing system based on underwater visible light communication. Asia-Pacific Congress on Sports Technology (APCST). 2013 \& Procedia Engineering (Elsevier). 2013; 60: 34-39.

[26] Stamm A, James DA, Thiel DV. Velocity profiling using inertial sensors for freestyle swimming. Sports Eng. 2013; 16: 1-11. https ://doi.org/10.1007/s12283-012-0107-6

[27] Gonzalo RD, Lemkaddem A, Renevey P, et al. Real-time monitoring of swimming performance. IEEE EMBS Conf. 2016: 4743-4746. 
[28] Dadashi F, Crettenand F, Millet GP, et al. Automatic front-crawl temporal phase detection using adaptive filtering of inertial signals. Journal of Sports Sciences. 2013; 31(11): 1251-1260. PMid:23560703. https://doi.org/10.1080/02640414.2013.778420

[29] Wang Z, Wang J, Zhao H, et al. CanoeSense: Monitoring canoe sprint motion using wearable sensors. IEEE Int. Conf. Systems, Man, and Cybernetics (SMC). 2016: 644-649.

[30] Jiao L, Bie R, Wu H, et al. Golf swing classification with multiple deep convolutional neural networks. Int. Journal of Distributed Sensor Networks. 2018; 14(10): 1-17.

[31] Groh BH, Fritz J, Deininger M, et al. Unobtrusive and wearable landing momentum estimation in ski jumping with inertial-magnetic sensors. Int. Conf. on Wearable and Implantable Body Sensor Networks (BSN). 2018: 102-105.

[32] Wang Z, Li J, Wang J, et al. Inertial sensor-based analysis of equestrian sports between beginner and professional riders under different horse gaits. IEEE Trans. on Instrumentation and Measuremen. 2018; 67(11): 2692-2704. https://doi.org/10.1109/TIM.2018.282 6198

[33] Wang Z, Guo M, Zhao C. Badminton stroke recognition based on body sensor networks. IEEE Trans. on Human-Machine Systems. 2016; 46(5): 769-775. https ://doi.org/10.1109/THMS . 2016. 2571265

[34] Sommer M, Hager C, Ronnqvist L. Synchonized metronome training induces changes in the kinematic properties of the golf swing. Sports Biomechanics. 2014; 13(1): 1-16. PMid:24968507. https : //doi.org/10.1080/14763141.2013.873817

[35] Zhang Z, Li S, Wan B, et al. The influence of X-Factor (trunk rotation) and experience on the quality of the badminton forehand smash. Journal of Human Kinetics. 2016; 53(1): 9-22. PMid:28149406. https ://doi.org/10.1515/hukin-2016-0006
[36] Skublewska-Paszkowska M, Montusiewicz J, Lukasik E, et al. Motion capture as a modern technology for analysing ergometer rowing. Advances in Science and Technology Research Journal. 2016; 10(29): 132-140. https://doi.org/10.12913/22998624/61941

[37] Zecha D, Greif T, Lienhart R. Swimmer detection and pose estimation for continuous stroke rate estimation. Multimedia Content Access: Algorithms and Systems VI, part of IS\&T/SPIE Electronic Imaging. 2012.

[38] Mara J, Morgan S, Pumpa K, et al. The accuracy and reliability of a new optical player tracking system for measuring displacement of soccer players. Int. Journal of Computer Science in Sport. 2017; 16(3): 175-184. https://doi.org/10.1515/ijcss-2017-0013

[39] Ahmed A, Asawal M, Khan MJ, et al. A wearable wireless sensor for real time validation of bowling action in cricket. IEEE Int. Conf. on Wearable and Implantable Body Sensor Networks (BSN). 2015: 1-5.

[40] Available from: https://eandt.theiet.org/content/artic les/2017/09/the-sensor-technology-powering-smart-c ricket-bats/

[41] Haykin S. Kalman Filtering and Neural Networks. Publisher: WileyInterscience; 2001.

[42] Gravina R, Alinia P, Ghasemzadeh H, et al. Multi-sensor fusion in body sensor networks: State-of-the-art and research challenges. Information Fusion. 2017; 35: 68-80. https ://doi.org/10.1016/ j.inffus.2016.09.005

[43] Zafar AZ, Hazry D. A simple approach on implementing IMU sensor fusion in PID controller for stabilizing quadrator flight control. Proc. IEEE Conf. Signal Processing and Its Applications. 2011: 28-32.

[44] Arndt C, Brefeld U. Predicting the future performance of soccer players. Statistical Analysis and Data Mining: The ASA Data Science Journal. 2016; 9: 373-382. https ://doi .org/10.1002/sam. 11 321 